Negative results

\title{
Evaluation of the interaction between LRRK2 and PARK16 loci in determining risk of Parkinson's disease: analysis of a large multicenter study
}

Lisa Wang a, Michael G. Heckman ${ }^{\mathrm{b}}$, Jan O. Aasly ${ }^{\mathrm{c}}$, Grazia Annesi ${ }^{\mathrm{d}}$, Maria Bozi ${ }^{\mathrm{e}, \mathrm{f}}$, Sun Ju Chung ${ }^{\mathrm{g}}$, Carl Clarke ${ }^{\mathrm{h}, \mathrm{i}}$, David Crosiers ${ }^{\mathrm{j}, \mathrm{k}}$, Gertrud Eckstein ${ }^{\mathrm{l}}$, Gaetan Garraux ${ }^{\mathrm{m}}$, Georgios M. Hadjigeorgiou $^{\mathrm{n}}$, Nobu Hattori ${ }^{\mathrm{o}}$, Beom Jeon ${ }^{\mathrm{p}}$, Yun J. Kim ${ }^{\mathrm{q}}$, Masato Kubo ${ }^{\mathrm{r}}$, Suzanne Lesage ${ }^{\mathrm{s}}$, Juei Jueng Lin ${ }^{\mathrm{t}, \mathrm{u}}$, Timothy Lynch ${ }^{\mathrm{v}}$, Peter Lichtner ${ }^{\mathrm{l}}$, George D. Mellick ${ }^{\mathrm{w}}$, Vincent Mok $^{\mathrm{x}}$, Karin E. Morrison ${ }^{\mathrm{y}}$, Aldo Quattrone ${ }^{\mathrm{d}, \mathrm{z}}$, Wataru Satake ${ }^{\mathrm{aa}}$, Peter A. Silburn ${ }^{\mathrm{bb}}$, Leonidas Stefanis ${ }^{\mathrm{f}, \mathrm{cc}}$, Joanne D. Stockton ${ }^{\mathrm{dd}}$, Eng King Tan ${ }^{\text {ee,ff }}$, Tatsushi Toda $^{\text {aa }}$, Alexis Brice ${ }^{\mathrm{s}}$, Christine Van Broeckhoven ${ }^{\mathrm{j}}$, Ryan J. Uitti ${ }^{\text {gg }}$, Karin Wirdefeldt $^{\text {hh }}$, Zbigniew Wszolek $^{\text {gg }}$, Georgia Xiromerisiou ${ }^{\mathrm{n}}$, Demetrius M. Maraganore ${ }^{\mathrm{ii}}$, Thomas Gasser ${ }^{\mathrm{jj}}$, Rejko Krüger ${ }^{\mathrm{jj}, \mathrm{kk}, \mathrm{ll}}$, 'Matthew J. Farrer ${ }^{\mathrm{mm}}$,

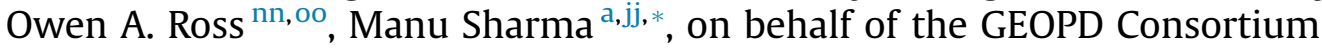

${ }^{a}$ Centre for Genetic Epidemiology, Institute for Clinical Epidemiology and Applied Biometry, University of Tuebingen, Tuebingen, Germany

${ }^{\mathrm{b}}$ Division of Biomedical Statistics and Informatics, Mayo Clinic, Jacksonville, FL, USA

${ }^{\mathrm{c}}$ Department of Neurology, St Olavs Hospital and NTNU, Trondheim, Norway

${ }^{\mathrm{d}}$ Institute of Molecular Bioimaging and Physiology, National Research Council, Catanzaro, Italy

e Neurology Department, Psychiatry Athens Hospital, Athens, Greece

${ }^{\mathrm{f}}$ 2nd Neurology Clinic, University of Athens, Attikon Hospital, Athens, Greece

${ }^{g}$ Department of Neurology, Asan Medical Center, University of Ulsan College of Medicine, Seoul, Korea

${ }^{\mathrm{h}}$ School of Clinical and Experimental Medicine, College of Medical and Dental Sciences, University of Birmingham, Birmingham, UK

${ }^{i}$ Institute of Clinical Sciences, College of Medical and Dental Sciences, University of Birmingham, Birmingham, UK

${ }^{\mathrm{j}}$ Neurodegenerative Brain Diseases Group, Department of Molecular Genetics, VIB, Antwerp, Belgium

${ }^{\mathrm{k}}$ Department of Neurology, University Hospital Antwerp, Antwerp, Belgium

${ }^{1}$ Helmholtz Zentrum München, German Research Centre for Environmental Health (Gmbh), Neuherberg, Germany

${ }^{\mathrm{m}}$ Department of Neurology, Cyclotron Research Centre, University of Liège, Liège, Belgium

${ }^{\mathrm{n}}$ Department of Neurology, Faculty of Medicine, University of Thessaly and Institute of Biomedical Research and Technology, CERETETH, Larissa, Greece

${ }^{\circ}$ Department of Neurology, Juntendo University School of Medicine, Tokyo, Japan

${ }^{\mathrm{p}}$ Institute Department of Neurology, Seoul National University Hospital, Seoul, Korea

${ }^{\mathrm{q}}$ ILSONG Institute of Life Science, Hallym University, Hallym Institute of Translational Genomics and Bioinformatics, Department of Neurology, Hallym University Sacred Heart Hospital, Gangwon, Korea

${ }^{\mathrm{r}}$ Laboratory for Genotyping Development, RIKEN Center for Integrative Medical Sciences, Yokohama, Japan

sINSERM U 1127, CNRS UMR 7225, Sorbonne Universités, UPMC Univ Paris 06 UMR S 1127, Institut du Cerveau et de la Moelle épinière, ICM, Paris, France

${ }^{\mathrm{t}}$ Department of Neurology, Chushang Show-Chwan Hospital, Nantou, Taiwan

u Department of Neurology, Chung-Shan Medical University Hospital, Taichung, Taiwan

${ }^{\vee}$ The Dublin Neurological Institute at the Mater Misericordiae University Hospital, and Conway Institute, University College Dublin, Dublin, Ireland

${ }^{\mathrm{w}}$ Eskitis Institute for Cell and Molecular Therapies, Griffith University, Queensland, Australia

${ }^{x}$ Therese Pei Fong Chow Research Center for Prevention of Dementia, Faculty of Medicine, The Chinese University of Hong Kong, Department of Medicine $\mathcal{E}$ Therapeutics, Clinical Neurology Research Centre, The Chinese University of Hong Kong, Prince of Wales Hospital, New Territories, Hong Kong

${ }^{\mathrm{y}}$ Faculty of Medicine, University of Southampton, Southampton, UK

${ }^{2}$ Department of Medical Sciences, Institute of Neurology, University Magna Graecia, Catanzaro, Italy

aa Division of Neurology/Molecular Brain Science, Kobe University Graduate School of Medicine, Kobe, Japan

${ }^{\mathrm{bb}}$ University of Queensland Centre for Clinical Research, Herston, Australia

${ }^{\mathrm{cc}}$ Divisions of Basic Neurosciences and Cell Biology, Biomedical Research Foundation of Academy of Athens, Athens, Greece

dd Neurosciences Department, Queen Elizabeth Hospital Birmingham, University Hospitals Birmingham NHS Foundation Trust, Birmingham, UK

ee Department of Neurology, Singapore General Hospital, National Neuroscience Institute, Singapore

${ }^{\mathrm{ff}}$ Programme in Neuroscience and Behavior Disorders, Duke-NUS Graduate Medical School, Durham, NC, USA

${ }^{\mathrm{gg}}$ Department of Neurology, Mayo Clinic, Jacksonville, FL, USA

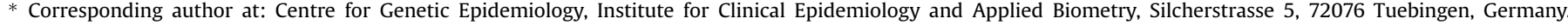
Tel.: +49 7071 2978259; fax: +497071295075.

E-mail address: manu.sharma@uni-tuebingen.de (M. Sharma).
} 
${ }^{\text {hh }}$ Department of Medical Epidemiology and Biostatistics, Karolinska Institute, Stockholm, Sweden

ii Department of Neurology, NorthShore University HealthSystem, Evanston, IL, USA

${ }^{\mathrm{ji}}$ Department of Neurodegenerative Diseases, Hertie Institute for Clinical Brain Science Research, University of Tuebingen, Tuebingen, Germany

${ }^{\mathrm{kk}}$ Clinical and Experimental Neuroscience, Luxembourg Centre for Systems Biomedicine, University of Luxembourg, Luxembourg City, Luxembourg

${ }^{11}$ Centre Hospitalier de Luxembourg (CHL), Luxembourg City, Luxembourg

${ }^{\mathrm{mm}}$ Djavad Mowafhagian Centre for Brain, University of British Columbia, Vancouver, British Columbia, Canada

${ }^{\mathrm{nn}}$ Department of Neuroscience, Mayo Clinic, Jacksonville, FL, USA

${ }^{\circ}$ School of Medicine and Medical Science, University College Dublin, Dublin, Ireland

\section{A R T I C L E I N F O}

Article history:

Received 30 June 2016

Received in revised form 27 September 2016

Accepted 28 September 2016

Available online 6 October 2016

Keywords:

Genetic epidemiology

Parkinson's disease

LRRK2

PARK16

\begin{abstract}
A B S T R A C T
A recent study MacLeod et al. has shown that an interaction between variants at the LRRK2 and PARK16 loci influences risk of development of Parkinson's disease (PD). Our study examines the proposed interaction between LRRK2 and PARK16 variants in modifying PD risk using a large multicenter series of PD patients (7715) and controls (8261) from sites participating in the Genetic Epidemiology of Parkinson's Disease Consortium. Our data does not support a strong direct interaction between LRRK2 and PARK16 variants; however, given the role of retromer and lysosomal pathways in PD, further studies are warranted.
\end{abstract}

(c) 2016 Elsevier Inc. All rights reserved.

\section{Introduction}

Genetic discoveries made over the years either by using linkage, array, and/or exome-based approaches have helped in advancing our knowledge of the genetic underpinnings of Parkinson's disease (PD) (International Parkinson Disease Genomics Consortium et al., 2011; Lesage and Brice, 2009; Trinh and Farrer, 2013). As we discover new loci relevant to idiopathic PD pathogenesis, it has become imperative to also understand the gene-gene interaction effect in modulating PD risk in population (see Supplementary Information) (Elbaz et al., 2011). Although the results of most gene-gene interactions studies in PD to date have pointed toward independent effects for PD susceptibility variants, an exception to this has been an assessment of functional-genetic interaction between the LRRK2 and PARK16 loci in which overexpression of $R A B 7 L 1$, a candidate gene for PARK16 locus, reversed the effects of the LRRK2 mutation and rescued the phenotypes (MacLeod et al., 2013). Therefore, this study aims to evaluate the interaction between several different LRRK2 and PARK16 variants in determining PD risk using a Caucasian series with more than 10,000 subjects from 14 different centers, and an Asian series with more than 5000 subjects from 5 different centers.

\section{Methods}

The Genetic Epidemiology of Parkinson's Disease (GEoPD) consortium includes investigators from 59 sites, across 30 countries and 6 continents, as of 2016. A total of 19 sites representing 17 countries and 4 continents agreed to contribute DNA samples and clinical data for the present study. In total, 15,976 subjects were included in this study, divided into a Caucasian series (5769 PD patients, 4988 controls) and an Asian series (1946 PD patients, 3273 controls). We selected 5 SNPs for the PARK16 locus (rs823139 [RAB7L1], rs708725 [RAB7L1], rs823156 [SLC41A1], rs11240572 [PM20D1], and rs708723 [RAB7L1]) because previously published studies suggested associations with PD risk and the respective sites also provided coverage of the PARK16 locus. We selected 2 SNPs from the LRRK2 gene (rs1491942, rs7133914) due to previously demonstrated associations with PD and minor allele frequencies high enough to allow for reasonable interaction analysis. Analysis was performed separately for the Caucasian series, the Asian series, and the combined series. We evaluated single variant associations using fixed effects logistic regression models adjusted for GEoPD site. Pairwise multiplicative interactions between LRRK2 and PARK16 variants were also examined using fixed effects logistic regression models. In addition to including terms for the given 2 individual variants and their interaction, these models were adjusted for the individual GEoPD site. Odds ratios (ORs) and 95\% confidence intervals (CIs) were estimated. Subjects were coded as either 0 (absence of the minor allele) or 1 (presence of the minor allele) for each variant. Variants with a MAF of $10 \%$ or greater in both the Asian and Caucasian series were examined under an additive model, with the subject coded as $(0,1,2)$, depending on the number of copies of the minor allele. To account for the 10 tests of LRRK2-PARK16 interaction that were performed in each series (Caucasian, Asian, or combined), we utilized a Bonferroni correction for multiple testing separately in each series, after which 2-sided $p$-values of 0.005 or lower were considered as statistically significant. All statistical analyses were performed using $\mathrm{R}$ Statistical Software. The local Ethics Committee at each GEoPD site approved the study. All participants signed an informed consent.

\section{Results}

Of the 10 interactions that were examined between the PARK16 and LRRK2 variants, nonsignificant evidence of gene-gene interaction was observed between LRRK2 rs1491942 and PARK16 rs11240572 in the combined series (interaction OR: 0.97, 95\% CI: 0.74-1.01, $p=$ 0.07, Supplementary Table 1). PARK16 rs11240572 appeared to have no effect on PD risk for individuals with the common GG genotype for LRRK2 rs1491942, but a slight protective effect for those with GC and CC LRRK2 rs1491942 genotypes (see Supplementary Information). Investigating this further in the stratified data (Supplementary Table 6), we observed for noncarriers of PARK16 rs11240572, LRRK2 rs1491942 a statistically significant higher risk of PD development in the Caucasian and combined series (OR 1.17 and 1.15, $p$-value $<0.001$ ). However, after correcting for multiple testing, it no longer approached statistical significance under the interaction model There were no other noteworthy interactions between LRRK2 rs1491942 and PARK16 variants in any series (all interaction $p \geq 0.25$, Supplementary Tables 3-5), or between LRRK2 rs7133914 and PARK16 variants in the Caucasian series (all interaction $p \geq 0.096$, 
Supplementary Table 7). Interaction ORs ranged between 0.85 and 1.20 , which supports the lack of a biologically meaningful interaction by lack of a notable deviation from an OR of 1. Between-site heterogeneity in interaction effects was generally relatively low (ranges between $0 \%$ and $35 \%$ with most around $0 \%$ ), lending consistency to the lack of interaction. Models adjusted for age and gender using the subset of subjects with complete information and random effects models also produced similar results in gene-gene interaction analyses.

\section{Discussion}

The identification of genetic mutations in genes linked to familial forms of PD (e.g., LRRK2, VPS35, and DNAJC13), and genetic variability within the PARK16 locus in genome wide association studies strongly implicates the role of retromer and lysosomal pathway in PD pathogenesis (Heckman et al., 2014; Soto-Ortolaza et al., 2013). Therefore, to understand the impact of interaction in world-wide populations, we performed a large multicenter study to assess the genetic evidence of interaction between LRRK2 and PARK16 locus. The results of our study do not provide evidence of a genetic interaction between PARK16 and LRRK2 variants with regard to risk of PD. Of note, the directionality of effect estimates, albeit with a much weaker effect size observed in the present study, involving the specific LRRK2 rs1491942/PARK16 rs11240572 interaction, are in agreement with previously published findings. Genetic interaction studies are limited by sample size and power because the variable of focus in an interaction study is the presence of the genotype of interest for both variants, and this occurs much less frequently than in the individual variant genotypes.

Therefore, even with our large sample size, power is still limited to detect moderate to small gene-gene interaction effects. Although there was some degree of concordance between our interaction findings and those that were previously reported, our results were much weaker than the strong LRRK2-PARK16 interaction that was previously reported (Beilina et al., 2014; MacLeod et al., 2013). Even with the large GEoPD sample size, which we have accrued to perform the present study, we are likely underpowered to detect weaker interaction effects. In addition, lack of genetic interaction does not exclude the presence of cellular or functional interaction. However, such genetic studies will be critical if we are to understand the role of gene-gene interaction in disease susceptibility.

\section{Disclosure statement}

The institution of L. Wang and M. Sharma is financially supported by the Courage-PD, an EU Joint ProgrammeNeurodegenerative Disease Research (JPND) project. The project is supported through the following funding organizations under the aegis of JPND-www.jpnd.eu: the Medical Research Council, United Kingdom, the French National Research Agency, the German Bundesministerium für Bildung und Forschung, the Italian Ministry of Health/Ministry of Education, the Israeli Ministry of Health, the Luxembourgian National Research Fund, the Netherlands Organisation for Health Research and Development, the Research Council of Norway, the Portuguese Foundation for Science and Technology, the Spanish National Institute of Health Carlos III and Michael J. Fox Foundation. M. J. Farrer has a patent on genetic variability in LRRK2 and Parkinson's disease (US8409809, US8455243B2) and on LRRK2 mouse models subsequently developed with royalities paid. Nobutaka Hattori has been serving as an advisory board member for Boehringer Ingelheim, as a result of attending advisory board meetings he received personal compensation; he also has been serving as an advisory board member for FP Pharmaceutical
Company, by attending these advisory meetings he received personal compensation and has been consulting with Ohtsuska Pharmaceutical Company, Kyowa Hakko Kirin Pharmaceutical Company, GlaxoSmithKline, Novartis, and Schering-Plough, and when he attended these advisory board meetings where he received personal compensation. Dr Gasser holds a patent concerning the LRRK2 gene and neurodegenerative disorders.

\section{Acknowledgements}

The Mayo Clinic Jacksonville is a Morris K. Udall Center of Excellence in Parkinson's Disease Research (grant number P50 NS072187) and was supported by the gift from the family of Carl Edward Bolch, Jr., and Susan Bass Bolch (RJU, ZKW, OAR). Owen A. Ross, $\mathrm{PhD}$, acknowledges funding support from the National Institutes of Health (grant number R01 NS78086). M. J. Farrer reports grants from the Canadian Federal Government, Cunhill Foundation, BC Leading Edge Endowment, during the conduct of the study; also personal fees from Gentech, personal fees from Teva, outside the submitted work. B. Jeon has received funding for travel from GlaxoSmithKline Korea and Novartis Korea; and has received research support as PI from Norvartis, Boehringer Ingelheim, Ipsen Korea, the Korea Health 21 R\&D Project, Ministry of Health and Welfare, Republic of Korea (\#A030001), ABRC (Advanced Biometric Research Center), KOSEF (Korea Science and Engineering Foundation), Seoul National University Hospital, the Mr Chung Suk-Gyoo and Sinyang Cultural Foundation, and the Song Foundation. Dr Lynch has served on an advisory board for Biogen and Novartis and has received honoraria from Lundbeck, Biogen, and Boerhinger-Ingelheim, and received funding from the NIH (Grant number: 1(GG010270)). Demetrius M. Maraganore, MD acknowledges the National Institutes of Health for funding support (grant number R01ES10751). E. K. Tan is funded by National Medical Research Council (RIE 2015 PD TCR and STaR), Duke-NUS Graduate Medical School (non grant support) and has received honoraria from Novartis, Boehringer Ingelheim and GSK. R. J. Uitti has received research funding from the NIH, PARRF, PSG, Noscira, Inc, and Advanced Neuromodulation Systems, Inc. Dr. Wszolek and Dr. Uitti are partially supported by the NIH/NINDS P50 NS072187, NIH/NIA (primary) and NIH/NINDS (secondary) 1U01AG04539001A1, Mayo Clinic Center for Regenerative Medicine, Mayo Clinic Center for Individualized Medicine, Mayo Clinic Neuroscience Focused Research Team (Cecilia and Dan Carmichael Family Foundation, and the James C. and Sarah K. Kennedy Fund for Neurodegenerative Disease Research at Mayo Clinic in Florida), the gift from Carl Edward Bolch, Jr., and Susan Bass Bolch, The Sol Goldman Charitable Trust, and Donald G. and Jodi P. Heeringa. Funding for Prof. Gasser was provided by the German Center for Neurodegenerative Diseases (DZNE) through the CoEN initiative (www.coen.org), German Research Foundation (GA 402/18-1), Federal Ministry of Education and Research (031A430A and 01ED1406) and Michael J. Fox Foundation (PPMI project). He received speakers' honoraria from Novartis, Merck-Serono, Schwarz Pharma, Boehringer Ingelheim, and Valeant Pharma and royalties for his consulting activities from Cefalon Pharma and Merck-Serono. R. Krüger has received research grants of the German Research Council (DFG; KR2119/8-1), the Fritz Thyssen Foundation ((10.11.2.153, Germany) and the Fond National de Recherche (PEARL [FNR/P13/6682797/Krüger]; NCER-PD, Luxembourg), as well as speakers' honoraria and/or travel grants from Abbvie, St. Jude and Medtronic. Studies at individual sites were supported by a number of different funding agencies worldwide including; Italian Ministry of Health (Ricerca Corrente, Ricerca Finalizzata); the Swedish Parkinson Foundation (697/14); the Federal Ministry of Education and Research (BMBF, NGFNplus; 
01GS08134] (RK); the NGFNplus (Neuron-Parkinson-subproject 7) (SG); CHRU de Lille, UnivLille 2, Inserm; French Ministry PHRCs (1994/, 2002/1918, 2005/1914); Association France Parkinson (2005); Fondation de France 2004-013306; Fondation de la Recherche Médicale (2006); PPF (synucléothèque 2005-2009); the 2 Centres de Ressources Biologiques (IPL-Lille, CHRU-Lille) and its scientific committee (AD, MCCH, Philippe Amouyel, Florence Pasquier, Régis Bordet); funding from France-Parkinson Association (R16058DD) and the program "Investissement d'avenir" ANR10-IAIHU-06; the Swedish Research Council; the Swedish Society for Medical Research; the Swedish Society of Medicine; funds from the Karolinska Institutet, the National Institutes of Health and National Institute of Neurological Disorders and Stroke [grant numbers 1RC2NS070276, NS057567, and P50NS072187]; Mayo Clinic Florida Research Committee CR programs (MJF) (ZKW); the Geriatric Medical Foundation of Queensland (GDM); a career development award from the Volkswagen Foundation and from the Hermann and Lilly Schilling Foundation (CK); the Research Committee of University of Thessaly (Code: 2845); the Queensland Parkinson's Project: R.S Boyle and A Sellbach (Proncess Alexandra Hospital, Brisbane), J.D. O'Sullivan (Royal Brisbane and Women's Hospital Brisbane), G.T. Sutherland, G.A Siebert and N.N.W Dissanayaka (Eskitis Institute for Cell and Molecular Therapies, Griffith University, Nathan, QLD), Belgian Science Policy Office Interuniversity Attraction Poles program, Belgium; the Alzheimer Research Foundation (SAO-FRA), Belgium; the Flemish Government initiated Methusalem Excellence program, Belgium; the Research Foundation Flanders (FWO), Belgium; the Agency for Innovation by Science and Technology Flanders (IWT), Belgium and the University of Antwerp Research Fund, Belgium; The Practical Research Project for Rare/Intractable Diseases from Japan Agency for Medical Research and development, AMED and Grantin-Aid for Young Scientists A (25713015) from the Japan Society for the Promotion of Science, JSPS; and the Laboratory of Neurogenetics, Biomedicine Department, CERETETH, Larissa, Greece (Code: 01-04-207) (GH, ED).

\section{Appendix A. Supplementary data}

Supplementary data associated with this article can be found, in the online version, at http://dx.doi.org/10.1016/j.neurobiolaging. 2016.09.022.

\section{References}

Beilina, A., Rudenko, I.N., Kaganovich, A., Civiero, L., Chau, H., Kalia, S.K., Kalia, L.V., Lobbestael, E., Chia, R., Ndukwe, K., Ding, J., Nalls, M.A., International Parkinson's Disease Genomics Consortium; North American Brain Expression Consortium, Olszewski, M., Hauser, D.N., Kumaran, R., Lozano, A.M., Baekelandt, V., Greene, L.E., Taymans, J.M., Greggio, E., Cookson, M.R., 2014. Unbiased screen for interactors of leucine-rich repeat kinase 2 supports a common pathway for sporadic and familial Parkinson disease. Proc. Natl. Acad. Sci. U. S. A. 111, 2626-2631.

Elbaz, A., Ross, O.A., Ioannidis, J.P., Soto-Ortolaza, A.I., Moisan, F., Aasly, J., Annesi, G., Bozi, M., Brighina, L., Chartier-Harlin, M.C., Destée, A., Ferrarese, C., Ferraris, A., Gibson, J.M., Gispert, S., Hadjigeorgiou, G.M., Jasinska-Myga, B., Klein, C., Krüger, R., Lambert, J.C., Lohmann, K., van de Loo, S., Loriot, M.A., Lynch, T. Mellick, G.D., Mutez, E., Nilsson, C., Opala, G., Puschmann, A., Quattrone, A. Sharma, M., Silburn, P.A., Stefanis, L., Uitti, R.J., Valente, E.M., Vilariño-Güell, C., Wirdefeldt, K., Wszolek, Z.K., Xiromerisiou, G., Maraganore, D.M., Farrer, M.J. Genetic Epidemiology of Parkinson's Disease (GEO-PD) Consortium, 2011. Independent and joint effects of the MAPT and SNCA genes in Parkinson disease. Ann. Neurol. 69, 778-792.

Heckman, M.G., Elbaz, A., Soto-Ortolaza, A.I., Serie, D.J., Aasly, J.O., Annesi, G., Auburger, G., Bacon, J.A., Boczarska-Jedynak, M., Bozi, M., Brighina, L., ChartierHarlin, M.C., Dardiotis, E., Destée, A., Ferrarese, C., Ferraris, A., Fiske, B., Gispert, S., Hadjigeorgiou, G.M., Hattori, N., Ioannidis, J.P., Jasinska-Myga, B., Jeon, B.S., Kim, Y.J., Klein, C., Kruger, R., Kyratzi, E., Lin, C.H., Lohmann, K., Loriot, M.A., Lynch, T., Mellick, G.D., Mutez, E., Opala, G., Park, S.S., Petrucci, S. Quattrone, A., Sharma, M., Silburn, P.A., Sohn, Y.H., Stefanis, L., Tadic, V., Tomiyama, H., Uitti, R.J., Valente, E.M., Vassilatis, D.K., Vilariño-Güell, C., White, L.R., Wirdefeldt, K., Wszolek, Z.K., Wu, R.M., Xiromerisiou, G. Maraganore, D.M., Farrer, M.J., Ross, O.A. Genetic Epidemiology Of Parkinson's Disease (GEO-PD) Consortium, 2014. Protective effect of LRRK2 p.R1398H on risk of Parkinson's disease is independent of MAPT and SNCA variants. Neurobiol. Aging 35, 266.e5-266.e14.

International Parkinson Disease Genomics Consortium, Nalls, M.A., Plagnol, V., Plagnol, V., Hernandez, D.G., Sharma, M., Sheerin, U.M., Saad, M., SimónSánchez, J. Schulte, C. Lesage, S., Sveinbjörnsdóttir, S., Stefánsson, K. Martinez, M., Hardy, J., Heutink, P., Brice, A., Gasser, T., Singleton, A.B. Wood, N.W., 2011. Imputation of sequence variants for identification of genetic risks for Parkinson's disease: a meta-analysis of genome-wide association studies. Lancet 377, 641-649.

Lesage, S., Brice, A., 2009. Parkinson's disease: from monogenic forms to genetic susceptibility factors. Hum. Mol. Genet. 18, R48-R59.

MacLeod, D.A Rhinn, H., Kuwahara, T, Zolin, A Di Paolo, G, McCabe, B.D. Marder, K.S., Honig, L.S., Clark, L.N., Small, S.A., Abeliovich, A., 2013. RAB7L1 interacts with LRRK2 to modify intraneuronal protein sorting and Parkinson's disease risk. Neuron 77, 425-439.

Soto-Ortolaza, A.I., Heckman, M.G., Labbe, C., Serie, D.J., Puschmann, A. Rayaprolu, S., Strongosky, A., Boczarska-Jedynak, M., Opala, G., KrygowskaWajs, A., Barcikowska, M., Czyzewski, K., Lynch, T., Uitti, R.J., Wszolek, Z.K. Ross, O.A., 2013. GWAS risk factors in Parkinson's disease: LRRK2 coding variation and genetic interaction with PARK16. Am. J. Neurodegener. Dis. 2 287-299.

Trinh, J., Farrer, M., 2013. Advances in the genetics of Parkinson disease. Nat. Rev. Neurol. 9, 445-454. 